Conclusions: The program was based on and added to the existing psychiatric and psychological emergency service for victims of multiple fatality incidents, which was necessary and useful to lowering stress levels in victims suffering from ASR. Post-analysis recommends adding an emergency telephone line for the staff and to offer PFA to all services in the hospital. A follow-up, evaluating the mental state of the patients to see if this program was successful is recommended.

Keywords: acute stress reaction; civilian; hospital; psychological first aid; war

Prehosp Disaster Med

New Concepts in Terrorism and Non-Conventional Global Threats: Ethics and Terror Medicine

Dr. A. Rossodivita; ${ }^{1}$ Prof. A.Trufanov ${ }^{2}$

1. San Raffaele Hospital and Scientific Foundation, IRCCS, University of Medicine "Life and Health", Milan, Italy

2. Irkutsk State Technical University, Irkutsk, Russia

Introduction: After the 11 September 2001 attacks, it became clear that all countries could experience a masscasualty incident (MCI) caused by the use of non-conventional weapons. Repeated terrorist attacks worldwide, and the global threat of a possible non-conventional chemical, biological, radiological, or nuclear explosive attack (CBRNE) now are considered worldwide problems. The size of attack, the setting, the sophisticated level of planning and organization, and the method employed were completely unprecedented. The risk of a possible CBRNE attack such as the risk of an attack using weapons of mass destruction (WMDs) is increasing. Public institutions such as government facilities, hospitals, universities, schools, or public gathering places may be targeted by terrorists using WMDs. All government and public institutions must be prepared to prevent or respond to such attacks. The health system plays a crucial role when reacting to terrorism. Appropriate, alternative actions for the response to these threats require planning with consideration of the level of risk and the local reality.

Methods: The authors analyzed and compared different forms of terrorism and non-conventional threats, with a specific analysis of the new form of international terrorism, in particular in the last decade. The study and application of laws and regulations based on protecting the population and with the respect to civil liberties, suggesting new concepts in terrorism and non-conventional threats, and terror medicine will be discussed.

Results: The knowledge of new terrorism concepts could help the international community improve responses and planning, teaching and drills, and improve preparedness in terror medicine. In addition, inter- and multi-level and interconnected cooperation at the national and international levels are emphasized.

Keywords: global; non-conventional weapon; terrorism; threat Prebosp Disaster Med
Fire Victims: A Useful Model of Multiple Injuries in Training for War or Terrorist Causalities

Colonel Francis Levy, $M D, P b D$

Chief Medical Officer, Service Départemental d'Incendie et de Secours 7, Colmar,France

A civilian fire victim is potentially hurt by three types of injuries: (1) disorders from explosion, falls, defenestration, etc.; (2) heat trauma from burns; and/or (3) toxic aggression from smoke inhalation and soot contamination. In large fires, there also is a probability of a greater number of victims requiring an incident command system, triage, or the evacuation of a population (especially in forest fires). The treatment of fire victims needs prehospital triage, decontamination, antidotes particularly for cyanide, and a heavy need for of transportation including oxygen stocks. Hospitals require intensive care units, burn center beds, and in the case of multiple victims, international cooperation. These fire situations can be used as a model for chemical, biological, radiological, and nuclear terrorist attacks because they also combine all the different threats and the same problems.

This presentation will demonstrate, with different examples, how the management of civil fire victims can be used as a lesson for war or terrorist attacks involving explosion, air toxins, and multiple traumas.

Keywords: burn patient; chemical, biological, radiological, and nuclear; fire victims; management

Prehosp Disaster Med

\section{Comparison of Injuries due to Terrorism and War}

Dena H. Jaffe; ${ }^{1}$ Kobi Peleg ${ }^{1,2}$

1. National Center for Trauma and Emergency Medicine Research, The Gertner Institute for Epidemiology and Health Policy Research, Tel-Hashomer, Israel

2. Department of Disaster Medicine, School of Public Health, TelAviv University, Tel-Aviv, Israel

Introduction: Injuries due to terrorism and war are not necessarily comparable, especially among civilians and military personnel. The authors sought evidence-based data for use in identifying gaps and establishing protocol for the management of injuries according to conflict type and population group.

Methods: A retrospective study was performed using hospitalization data from the Israel National Trauma Registry (November 2000-December 2006).

Results: Terrorism and war accounted for trauma hospitalizations among 1,784 civilians and 802 military personnel. Most civilians (93\%) were injured in acts of terrorism and transferred to trauma centers by land. Critical injuries and injuries to multiple body regions were more likely in terrorism than war. In contrast, military personnel were injured in both acts of terrorism and war and brought to trauma centers by land and air. Among military personnel war injuries tended to be less severe than those due to terrorism. Rates of first admission to orthopedic surgery were greater for all casualties except for civilians injured by acts of terrorism who were equally likely to be admitted to the intensive care unit. The rate of $\geq 1$ surgical procedure within 12 hours of admission was higher among victims of terrorism than war casualties. In-hospital mortality was higher 